\title{
DEMO physics challenges beyond ITER
}

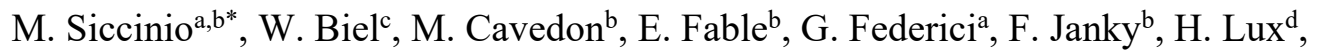

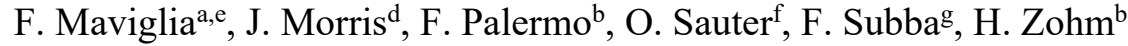 \\ ${ }^{a}$ EUROfusion Consortium, Garching bei München, Germany \\ ${ }^{b}$ Max-Planck-Institut für Plasmaphysik, Garching bei München, Germany \\ ${ }^{c}$ Forschungszentrum Jülich, Jülich, Germany \\ ${ }^{d}$ Culham Centre for Fusion Energy, Abingdon, United Kingdom \\ ${ }^{e}$ Associazione Euratom-ENEA sulla Fusione, Centro Ricerche Frascati, Frascati, Italy \\ fÉcole polytechnique fédérale de Lausanne (EPFL), Swiss Plasma Centre (SPC), Lausanne, Switzerland \\ ${ }^{g}$ NEMO Group, Politecnico di Torino, Turin, Italy
}

\begin{abstract}
For electricity producing tokamak fusion reactors like EU-DEMO, it is prudent to choose a plasma scenario close to the ITER baseline, where the largest amount of experimental evidence is available. Nevertheless, there are some aspects in which ITER and EU-DEMO have to differ, as the simple exercise of up-scaling from ITER to a larger device is constrained both by physical nonlinearities and by technological limits. In this work, relevant differences between ITER and the current EU-DEMO baseline in terms of plasma scenario are discussed. Firstly, EU-DEMO is assumed to operate with a very large amount of radiative power originating both from the scrape-off layer and, markedly, from the core. This radiation level is obtained by means of seeded impurities, whose presence significantly affects many aspects of the scenario itself, especially in terms of transient control. Secondly, because of the need of breeding tritium, the EU-DEMO wall is less robust than the ITER one. This implies that every offnormal interruption of the plasma discharge, for example in presence of a divertor reattachment, cannot rely on fastshutdown procedures finally triggering a loss of plasma control at high current, but other strategies need to be developed. Thirdly, the ITER method for the control of the so-called sawteeth (ST) has been shown to be too expensive in terms of auxiliary power requirements, thus other solutions have to be explored. Finally, the problem of actively mitigating, or suppressing, the Edge Localised Modes (ELMs) has recently increased the interest on naturally ELM-free regimes (like QH-mode, I-mode, and also negative triangularity) for EU-DEMO, thus increasing the needs for ELM mitigation or suppression with respect to the approach adopted in ITER.
\end{abstract}

Keywords: EU-DEMO, ITER, plasma scenario, ELM-free regimes, ELMy H-mode

\section{Introduction}

In the European Research Roadmap to the Realisation of Fusion Energy [1], the ITER's role is acknowledged as crucial. ITER will be in fact the first machine demonstrating the generation of fusion power at a level greatly exceeding the external heating power needed to sustain the plasma. Also, ITER will be the first device allowing for the exploration of plasma conditions which are not accessible in present machines (e.g. the simultaneous achievement of high density and low collisionality, or dominantly alpha heated plasmas). For this reason, the most natural choice for the design of a EU-DEMOnstration [2] fusion reactor consists of assuming the ITER baseline as the starting point for the scenario definition, since no other scenario is likely to have such robust experimental evidence on support in reactor relevant conditions. The target fusion gain of ITER ( $Q=10$, where $Q$ is the ratio between the generated fusion power, $P_{f u s}$, and the power injected in the plasma $P_{a u x}$ ) can however be shown to be insufficient for a net electric power production, in view of the non-unitary efficiencies associated both to the thermodynamic Rankine cycle and the H\&CD wall plug. Thus, an up-scaling from ITER - in terms of major radius, or magnetic field, or both - is unavoidable for the realization of an EU-DEMO with an "ITER-like" scenario. The extrapolation from ITER to EU-DEMO exhibits nevertheless strong nonlinearities, due to plasma physics and technology. Besides, the differences in ITER and EU-DEMO missions introduce further design constraints, so that some technical solutions adopted in ITER might not be compatible with the availability requirements of EU-DEMO. As a matter of fact, ITER is still considered an experimental reactor, whereas, according to the Roadmap, EU-DEMO shall

- Demonstrate a net electricity production.

- Breed its fusion fuel (tritium).

- Exhibit a sufficiently high availability, and thus a long lifetime of its components, to show the ability of successive, commercial Fusion Power Plants (FPP) to play a role in the energy market.

In this work, some of the most significant deviations from the ITER baseline plasma scenario which have been introduced in EU-DEMO, as well as the reasons leading to their introduction, are discussed. This paper is structured as follows: in section 2, the main parameters of EU-DEMO latest baseline are reviewed, and compared to the corresponding ITER's ones. All the investigations presented in the following sections are based on such baseline, unless stated otherwise. In section 3 , the role of core impurity radiation is analyzed. In section 4, the Plasma Facing Components (PFC) 
protection strategy in presence of divertor reattachment in the two devices is discussed, whereas section 5 deals with the control of the sawteeth. Section 6 discusses the consequences of the adoption of naturally ELM-free scenarios in EU-DEMO, and, finally, conclusions are drawn in section 7 .

\section{EU-DEMO 2018 Baseline}

In spring 2018, a new baseline for EU-DEMO produced by the systems code PROCESS [3,4] was released. With the name "baseline" is here indicated a design point consistent with a number of physics and technology constraints and determined by means of a systems code, which contains a number of simplified physics and technology models. Here, the top-level requirements of $2000 \mathrm{MW}$ fusion power and $500 \mathrm{MW}$ plant net electric output power as well as 2 hours burn time have been maintained from the previous baselines, see e.g. [5].

\begin{tabular}{lll}
\hline & EU-DEMO & ITER \\
& 2018 & \\
\hline$R[\mathrm{~m}]$ & 9.00 & 6.2 \\
$A$ & 3.1 & 3.1 \\
$B_{0}[\mathrm{~T}]$ & 5.86 & 5.3 \\
$q_{95}$ & 3.89 & 3 \\
$\delta_{95}$ & 0.33 & 0.33 \\
$\kappa_{95}$ & 1.65 & 1.7 \\
$I_{p}[\mathrm{MA}]$ & 17.75 & 15 \\
$f_{N I}$ & 0.39 & $\sim 0.2$ \\
$f_{C D}$ & $>5 \%$ & $5-10 \%$ \\
$P_{f u s}[\mathrm{MW}]$ & 2000 & 500 \\
$P_{\text {sep }}[\mathrm{MW}]$ & 170.4 & 89 \\
$P_{L H}[\mathrm{MW}]$ & 120.8 & 52 \\
$H_{98}$ & 0.98 & 1 \\
$<n>/ n_{G W}$ & 1.2 & $\sim 1$ \\
$<T>[\mathrm{keV}]$ & 12.49 & 8.9 \\
$\beta_{N}[\% \mathrm{mT} / \mathrm{MA}]$ & 2.5 & 1.8 \\
$Z_{\text {eff }}$ & 2.12 & 1.78 \\
$P_{\text {sep }} B / q_{95} A R$ & 9.2 & 8.2 \\
{$[\mathrm{MW} \mathrm{T} / \mathrm{m}]$} & & \\
$P_{\text {sep }} / R[\mathrm{MW} / \mathrm{m}]$ & 18.9 & 14.35 \\
$\mathrm{Pulse}_{[\mathrm{sec}]}$ length & 7200 & 600 \\
\hline
\end{tabular}

Table 1. EU-DEMO Physics Baseline 2018 relevant machine parameters and corresponding values for ITER. EU-DEMO data have been produced with the systems code PROCESS.

Table 1 summarizes the most relevant (physics related) parameters of EU-DEMO 2018 for the flat-top plasma phase. Note that EU-DEMO is currently found in the pre-conceptual design phase [2], thus the baseline parameters shown here have not to be understood as a final design point, but rather as a starting point for the ongoing activities. The corresponding values of ITER 15 MA baseline scenario [6,7] are also reported for comparison. Both ITER and EU-DEMO exhibit a small fraction of auxiliary driven current $f_{C D}$. In EU-DEMO, in particular, there is no dedicated actuator for that, although some current drive may originate from preemptive NTM control and/or NB burn control (see next sections). Density and temperature profiles of the 2018 EU-DEMO baseline, calculated with the transport code ASTRA $[8,9,10]$ are shown in Fig.1. As mentioned in the introduction, the reference EU-DEMO scenario is analogous to the ITER 15 MA baseline, both assuming a pulsed operation in ELMy H-mode [11] with a confinement time in line with the well-known IPB98(y,2) scaling [12]. There are however some aspects on which the two scenarios differ, although not immediately visible in the table above. These are analyzed in the following sections.

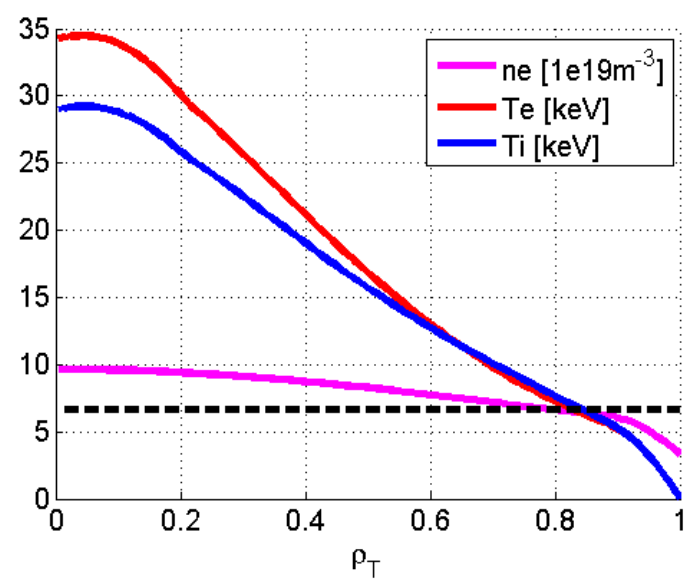

Fig 1. Density and temperature profiles for EU-DEMO baseline 2018 calculated with ASTRA. The horizontal dashed black line identifies $n=n_{G W}$, where $n_{G W}$ is the Greenwald density. The slightly non-monotonic behavior of the temperature profiles in proximity of the magnetic axis is just a numerical artifact, which however does not impact on the overall results. To produce this profiles, the values of $I_{p}, B_{0}$ and $q_{95}$ are kept the same as in PROCESS.

\section{Core Impurity Radiation}

One important point with respect to which ITER and EU-DEMO differ is the large amount of core radiation, which is necessary in EU-DEMO to protect the divertor and keep $P_{\text {sep }}$ reasonably close to $P_{L H}$. In ITER, the unavoidable synchrotron and bremsstrahlung losses reduce the power carried by charged particles across the separatrix from the $150 \mathrm{MW}$ of heating power $P_{\text {heat }}$ (corresponding to $100 \mathrm{MW} P_{\alpha}$ plus $50 \mathrm{MW} P_{\text {aux }}$ ) to about $80 \mathrm{MW}$, which can be dealt with by the divertor in presence of seeded SOL impurities, like e.g. Ne or $\mathrm{N}$ [13]. The situation is however different in EU-DEMO, where, in absence of additional core radiation, the power 
$P_{\text {sep }}$ would be larger than $350 \mathrm{MW}$, an amount which could not be radiated in the SOL without compromising the stability of the discharge. Thus, an high-Z impurity, e.g. Xe [14], is seeded in the EU-DEMO core (on top of the SOL seeded radiator, which is Ar), with the purpose of enhancing the overall fraction of power exhausted via radiation, which uniformly distributes on the very large first wall surface and does not concentrate on the small target wetted area.

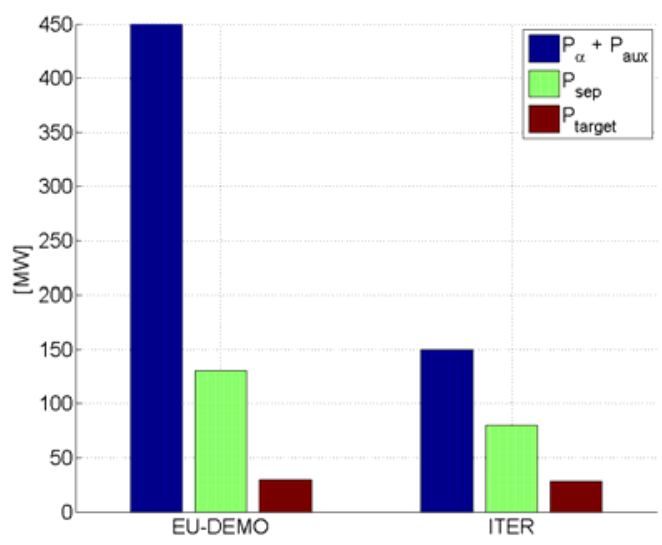

Fig. 2. Total heating power, power at the separatrix and maximum tolerable divertor power in ITER and EU-DEMO. Data for EU-DEMO are taken from PROCESS.

The energy flows for the two devices are depicted in Fig.2. The differences between $P_{\text {heat }}$ and $P_{\text {sep }}$, and between $P_{\text {sep }}$ and $P_{\text {target }}$, correspond to the core and SOL radiation amount, respectively. Fig. 3 shows the radiation power density profile corresponding to the ASTRA calculation in Fig.1. The Xe density is assumed to be radially constant, which leads to a hollow concentration profile.

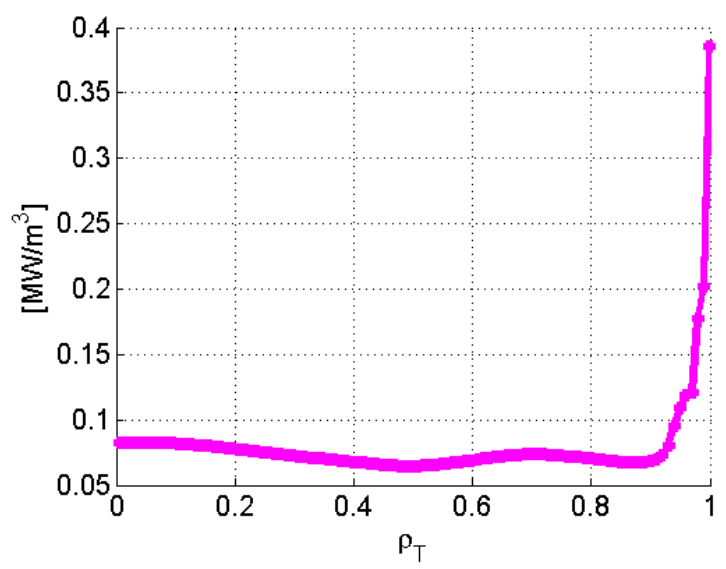

Fig. 3. Total radiation power density profile in EU-DEMO core. Synchrotron radiation, bremsstrahlung and line radiation from seeded impurities $(\mathrm{Xe})$ are considered, the latter being the dominant contribution. Most of the radiated power is localised in the pedestal region, where the $\mathrm{Xe}$ cooling factor is significantly higher.

As one can observe, the radiated power - which is mostly originating from the impurity line radiation, although also synchrotron and bremsstrahlung play a non-negligible role - is strongly localized in the plasma edge, where the temperature is favorable for $\mathrm{Xe}$ to radiate. Of course, $\mathrm{Xe}$ is expected to be present everywhere in the plasma core, but only in the plasma edge its cooling factor is sufficiently high to radiate significantly. Also, the edge region has a much larger volume than the central regions, this leading to a large overall radiated power.

The presence of a core radiator has nevertheless a strong impact on the plasma control, in view of the large residence time foreseen for such species in the confined plasma, which implies that no "fast" method exists to remove Xe from the core if radiation becomes excessive. In Fig.4, an example of EU-DEMO plasma control simulation produced with ASTRA/Simulink [15] is shown. This tool, starting from the profiles depicted in Fig. 1, is able to produce dynamical simulations and investigate the response of the plasma, as well as of the actuators, when a perturbation is applied [15]. At $t=$ $200 \mathrm{sec}$, the core radiation is artificially increased by introducing $1 \mathrm{mg} \mathrm{W}$ at about $\rho_{T}=0.7$. This has the effect of decreasing the plasma temperature, increasing in parallel also the total radiation from $\mathrm{Xe}-$ as, at lower temperature, the Xe average cooling factor throughout the plasma core increases. This accelerates the cooling of the plasma, leading to an unstable runaway situation. As one can observe, the total radiation from the core almost doubles in a few seconds.

Turning on about $100 \mathrm{MW}$ of additional auxiliary power is an insufficient measure to maintain the plasma hot and avoid a dangerous H-L transition, as shown in Fig.4. Currently, EU-DEMO assumes an installed H\&CD power of $130 \mathrm{MW}$ [16], but no final decision has been taken on which technologies (among EC, IC and NB) has to be present. All investigation concerning transients - e.g. ramp-up and down, not discussed in the present paper - are carried out within this limit. Afterwards, the plasma controller tries to recover the lost fusion power by increasing the pellet injection frequency, and enhance central density. This strategy is very effective, but also quite dangerous, as EU-DEMO is already operating close to the density limit (it is typically assumed [2,5,14] that EU-DEMO discharge can survive up to $\sim 1.2 n_{G W}$, with $1.3 n_{G W}$ being the upper acceptable value), and an excessive increase of the plasma density can fatally compromise the stability of the discharge. In Fig.4, the region where the density limit is violated (and thus the shown results are unphysical, and not happening in reality) has been shadowed in yellow.

Ensuring the controllability of the plasma in presence of plasma transients (planned and unplanned) turned out as one of the main difficulties encountered up to now in the design of the EU-DEMO plasma scenario. The presence of the impurities greatly enhances the complexity of the problem, which for the time being has not been fully solved. A careful balance between H\&CD actuators and pellets injection has to be individuated to make such offnormal transients treatable. 

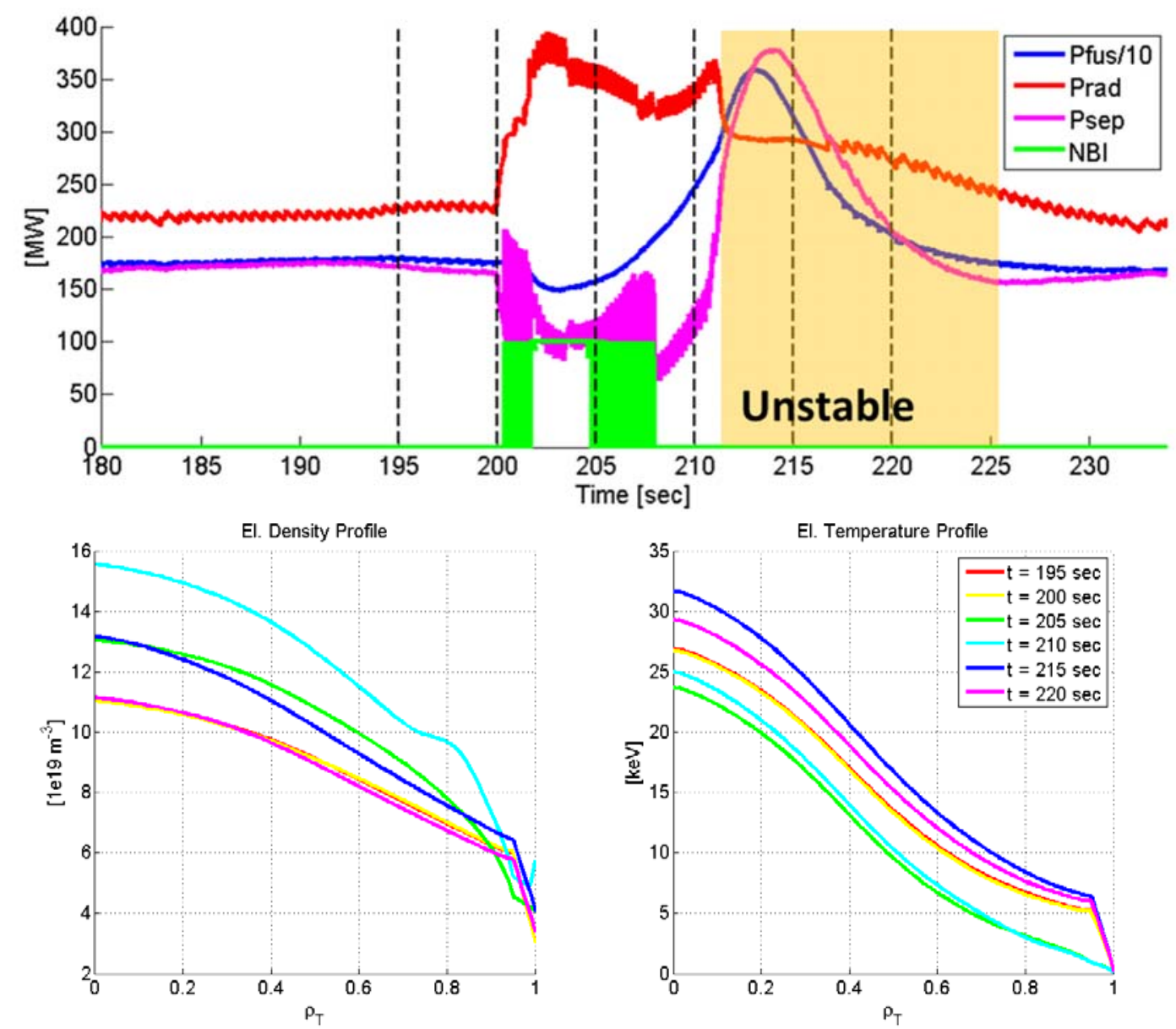

Fig. 4. Top: time traces of different power signals in presence of a radiative instability simulated with ASTRA/Simulink. The radiation was artificially increased in the numerical simulation by injecting $1 \mathrm{mg}$ of $\mathrm{W}$ at $t=200 \mathrm{sec}$. The region shaded in yellow shows where the Greenwald density limit (i.e. $n>1.3 n_{G W}$ - see main text) is largely violated, and thus the numerical results are no longer valid as the plasma is unstable. Bottom: radial density and temperature profiles at different times - marked by vertical dashed lines in the top figure. Note that the fusion power at the beginning of the simulation is lower than the $2 \mathrm{GW}$ indicated in Table 1 . This is due to the fact that ASTRA, at the same field and current as PROCESS, typically yields a lower fusion power (since different transport models are employed).

\section{Divertor Reattachment}

Because of the necessity of breeding tritium, the EUDEMO wall must be sufficiently thin to allow the fusion generated neutrons reaching the breeding region. The present first wall design foresees in fact a $\sim 3 \mathrm{~mm}$ metal layer (W and EUROFER) between the vacuum chamber and the coolant (water or He) $[17,18]$. Such weak wall cannot withstand a contact with the plasma, unless the stored (kinetic and magnetic) energy is extremely low at least a factor of 10 below the flat-top values. Even the foreseen sacrificial limiters for the wall protection during incidental plasma-wall contacts [19] would be damaged if $I_{p}>\sim 5 \mathrm{MA}$, whereas $I_{p} \approx 20 \mathrm{MA}$ during the flattop phase. Thus, any emergency plasma shutdown procedure which relies on a plasma/wall contact, or in general on a loss of plasma control at high current, is not considered viable, as the consequences for the first wall might be too severe. This implies that EU-DEMO requires the definition of strategies to ensure the integrity of all plasma-facing components during the current rampdown when an emergency plasma termination takes place.

A typical situation where such emergency shutdown is needed is the loss of plasma detachment at the divertor. Both ITER and EU-DEMO are operated with a (partially or fully) detached divertor, the heat flux on the target plates exceeding the technological limit of $\sim 10 \mathrm{MW} / \mathrm{m}^{2}$ otherwise [20]. It has been elsewhere shown [21] that if detachment is lost in the current ITER-like lower single null divertor configuration of EU-DEMO, the heat flux to the coolant reaches the critical heat flux in about three seconds if no countermeasures are adopted. In EUDEMO, the foreseen countermeasure is currently represented by divertor sweeping, which has been illustrated in [21,22] and which is able to let the target survive for some tens of seconds in presence of 
reattachment. This time lapse is required to ramp-down the plasma current without control losses or safely return to the detached state. Dedicated simulations carried out with the code RAPTOR [23] have in fact pointed out that, even by optimizing the plasma shape during the ramp as suggested in [24], the plasma current cannot be ramped down faster than $\sim 0.1 \mathrm{MA} / \mathrm{sec}$ - at best without losing the vertical stability of the plasma column. This is shown in Fig.5, where the stable region of the ramp trajectory is identified in terms of the internal inductance defined as in [12]. Incidentally, this result has of course also an impact on the planned rampup and down rates, which have to be lower than $\sim 0.05$ $\mathrm{MA} / \mathrm{sec}$ unless further shape adjustments are possible. The situation is different in ITER. In spite of the fact that disruptions - and plasma/wall contacts in general - are a very serious issue which must occur at the lowest possible rate [25] and which require a reliable mitigation system, an abrupt discharge interruption via matter injection is one of the viable strategies for the fast termination of the plasma. The ITER first wall, which has no requirements related to tritium breeding, is more robust, and thus a certain number of disruptive events is considered acceptable during the device lifetime [26]. Also, divertor sweeping coils are not included in the machine design.

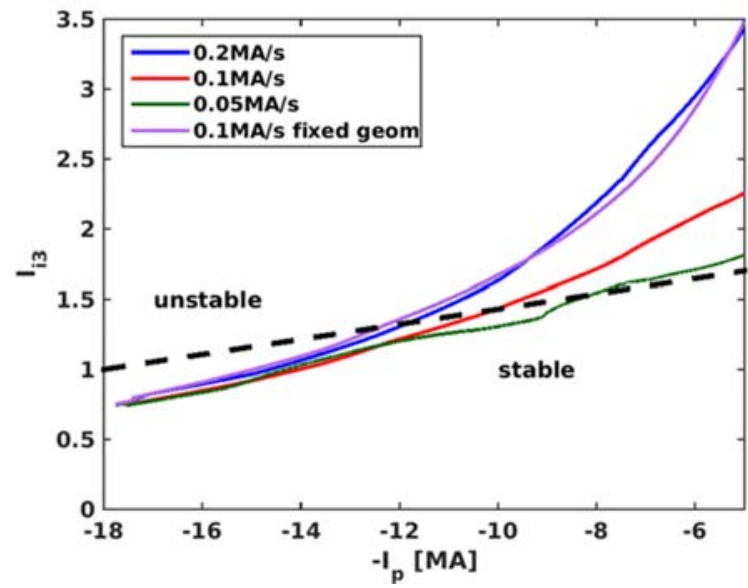

Fig. 5. Evolution of the internal inductance $l_{i 3}$ during rampdown phases assuming different rates evaluated with RAPTOR [23]. For the case with $100 \mathrm{kA} / \mathrm{s}$ rate, also the curve at fixed plasma geometry has been added. From the vertical stability analyses with standard profiles, values of $l_{i 3}$ above the dashed line cannot be controlled.

As divertor sweeping in EU-DEMO must be already active when the transition from attachment to detachment begins, it is necessary to install diagnostics able to detect a loss of plasma detachment at its onset, or even before it occurs (a continuous sweeping during the entire discharge in normal operation is not viable, as the $\mathrm{AC}$ losses in the coils would be too large to be dealt with [22]). For these reasons, the feasibility of a detachment control based on visible and UV spectroscopy is currently investigated. Spectroscopic measurements in different regions of the divertor have been largely used to monitor the status of detachment given their relatively easy implementation (for a review see [27]). More recently, the ratio of emission lines of nitrogen from different and equal ionization stages could be used to characterize the detachment evolution [28,29]. Possibly, such method can be extended to other impurities, e.g. Ar, which is at the moment the selected seeded SOL radiator for EU-DEMO. Along the same line, the ratio of Balmer-lines has been employed to measure the plasma recombination fraction along the outer divertor leg [30]. Based on these methods, a control signal for the divertor detachment with spectroscopic measurements might be possible. Fig. 6 shows an example of different radiation distribution in two SOLPS fluid cases in the transition from attached to detached divertor. The formation of a highly radiative region in front of the outer divertor plate, which should be detected by the diagnostics, is noticeable.

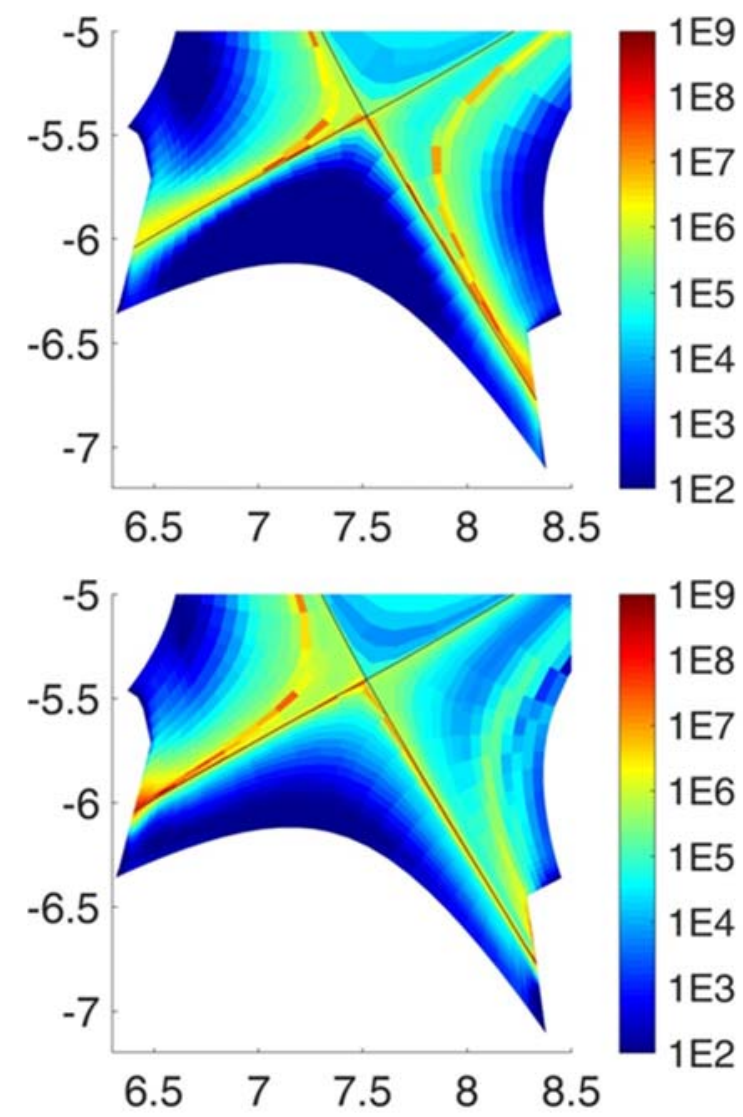

Fig. 6. Total radiation density $\left(\mathrm{W} / \mathrm{m}^{3}\right)$ in a barely detached case (top - with pressure ratio upstream to target close to one) and in a more strongly detached case (bottom - with pressure ratio $\sim 4$ ) calculated with SOLPS.

\section{Sawteeth Control}

Although the presence of sawteeth (ST) might also have some beneficial aspects, for example increasing $\mathrm{He}$ flushing from the centre of the plasma, an uncontrolled crash is very likely to trigger Neoclassical Tearing Modes (NTMs), which in turn risk initiating chains of events eventually leading to disruptions. The presence of a large, stabilizing fast particle population - fusion born alphas - is expected to significantly increase the ST period, which in turn implies a large amplitude (and 
therefore more dangerous) ST crash. Preliminary estimates carried out following the empirical method proposed in [31] (and shown in Fig.7) show that, in EUDEMO, an acceptable ST period to reduce the risk of NTM onset after the crash should be about 5-10 times shorter than the natural period. It is therefore to be expected that a ST control strategy based on destabilization of the mode until the required frequency (and amplitude) is obtained, as foreseen in ITER, would be particularly demanding in terms of auxiliary heating. For this reason, EU-DEMO must pursue a pacing of the ST rather than their destabilization - i.e. by means of $\mathrm{CD}$, the ST crash is avoided by stabilizing the kink for a certain period of time.

When CD is turned off, a ST crash under controlled conditions occurs. In parallel, a pre-emption of the NTMs is undertaken, in order to avoid their triggering after the ST-crash (see a more detailed description of this method in [32]). In Fig.8, simulations carried out with ASTRA show that keeping ST stable for several minutes allows a reduction in the required $\mathrm{H} \& \mathrm{CD}$ power up to a factor 3 compared to the ITER-like destabilization approach. At this stage, the optimum duration of the ST pacing has not been assessed yet. In these simulations, the stability of the ST is modelled by employing the well-known critical shear criterion proposed by Porcelli et al. [33]. Currently, the ST control power requirement for the H\&CD system design is set to $30 \mathrm{MW}$ of ECCD. emption phase to the whole discharge, thus avoiding any active control of the ST, are also considered.

The situation is much less critical in ITER, as can be observed in Fig.7. This is due not only to the fact that the alpha particle population is not as large as in EU-DEMO, but also because $\beta_{N}$ is significantly lower (about 1.8 against $\sim 2.5$ in EU-DEMO - see Table 1). This reduces significantly the risk of NTM triggering after a ST crash, making the approach based on mode destabilization convenient.

A large ST crash has however the drawback of inducing significant oscillations in the fusion power and, in general, in the various loss channels. This is depicted in Fig.9, which shows an ASTRA/Simulink simulation of a large ST crash. Immediately after the ST crash itself, the power crossing the separatrix $P_{\text {sep }}$ exhibits a peak, due to the associated fast increase in transport. At this stage, it is not clear whether this could be safely dealt with by the divertor. This is subject of future analyses. Then, $P_{\text {sep }}$ decreases as a consequence of the reduced $P_{\text {fus }}$. The relevant time scale for the recovery of the original fusion power level is the current diffusion time, i.e. the time needed for the current profile to return to the original shape. In view of the high central temperatures, this evolution is quite slow in EU-DEMO, and several tens of seconds might be required to restore $P_{f u s}$.

Alternative approaches, employing for example IC [34], or extending the temporal duration of the NTM pre-

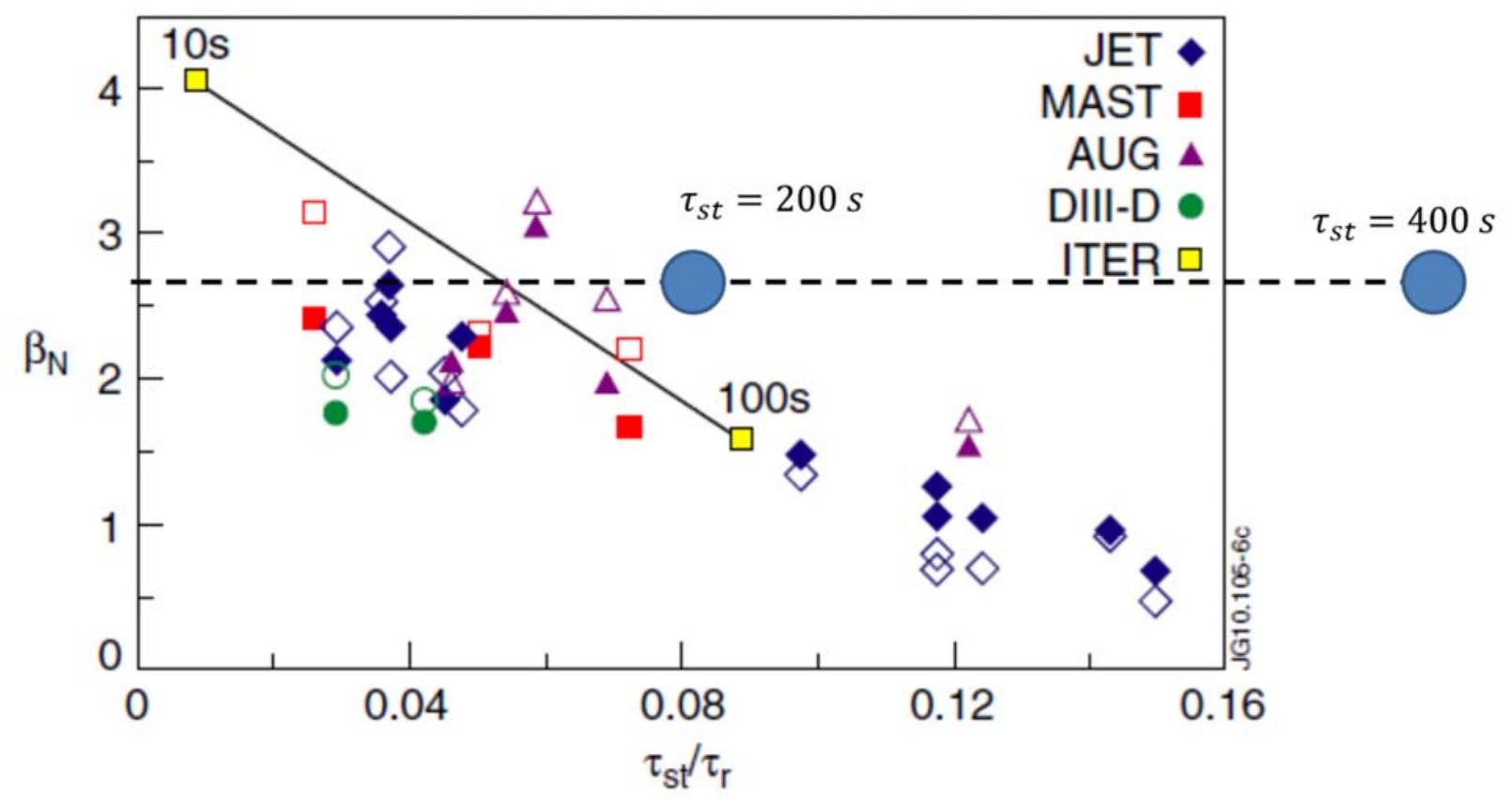

Fig. 7. Figure adapted from [31]. The experimental points identify the minimum $\beta_{N}$ for the onset of NTM after a ST crash as a function of the ST period (expressed in plasma resistive time $\tau_{r}$ units). For the DEMO target value of $\beta_{N}$, the required ST period should then be lower than $\sim 0.02 \tau_{r}$, whereas the expected natural period is a factor 5-10 larger, according to different estimates. Full symbols refer to experimental data, open symbols to an analytical model described in the referenced paper [31] 


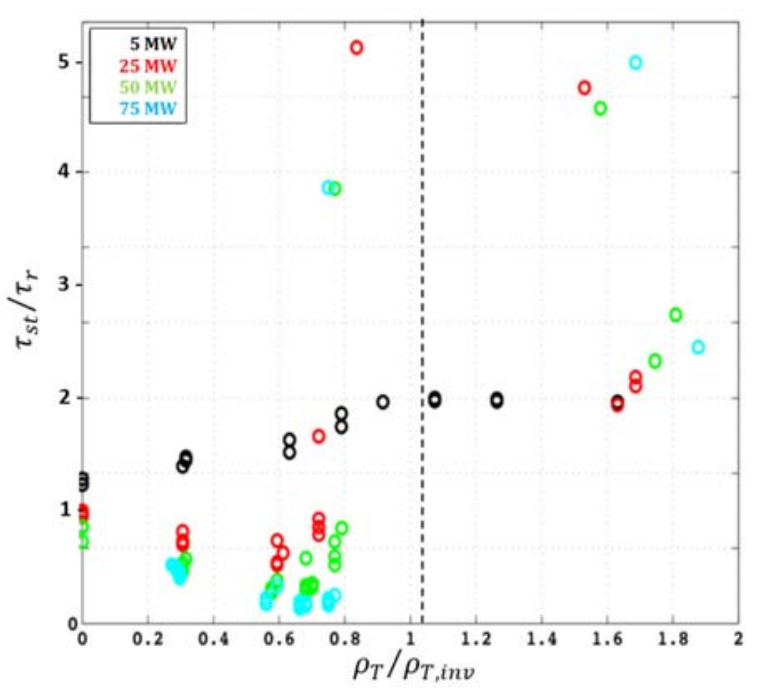

Fig. 8. Resulting ST period normalized to resistive time vs. position of the EC injection, normalized to the inversion radius coordinate. The EC power is identified by the color scale of the symbols. The vertical dashed line identifies the $q=1$ surface. At $q=1$, the resistive time $\tau_{r}$ amounts to $\sim 300 \mathrm{~s}$.

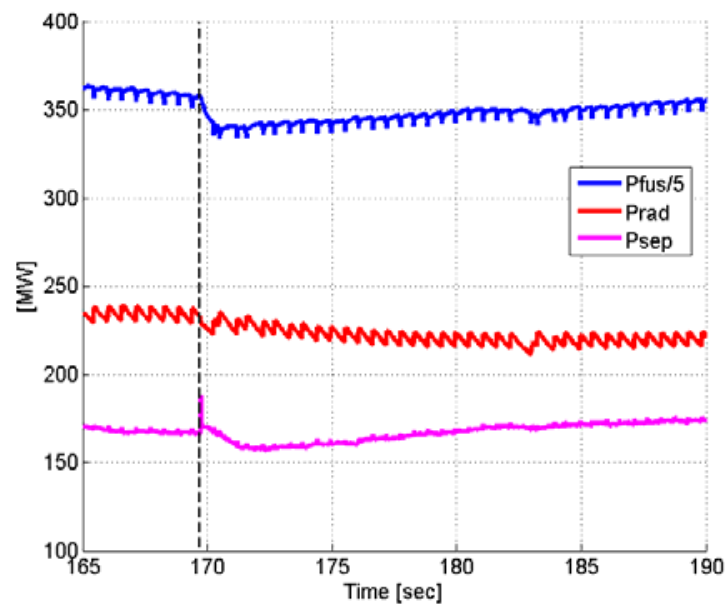

Fig. 9. Time traces for different power signals in presence of a large sawteeth simulated with ASTRA/Simulink. The ST occurs at $t=169.7 \mathrm{sec}$, immediately followed by a "spike" in the power crossing the separatrix. Small oscillations are due to pellet injections during plasma fuelling.

It is also important to note that the reduction of $P_{\text {sep }}$ might require the intervention of auxiliary heating, in order to avoid a pernicious H-L transition. However, the slow time scales at which it occurs (comparable with the confinement time $\tau_{E} \sim 3 \mathrm{sec}$ ) ensure a reasonable control margin.

\section{ELM-free regimes}

A simple estimate from a scaling recently suggested in [35] indicates that, in EU-DEMO, a natural type I Edge Localised Mode (ELM) releasing $10 \%$ of the pedestal energy would deposit the equivalent of $\approx 10 \mathrm{MJ}$ of energy in around one millisecond on the target plate. Simulations performed with the code RACLETTE [36] have shown that even a single ELM event of this kind will be sufficient to cause surface melting of the Wcoated target plate, and a few tens of these events will be able to ablate half of the total thickness of the $\mathrm{W}$ layer.

This risk poses a serious question mark on the suitability of $\mathrm{H}$-mode as a reactor scenario, since a reliability of $100 \%$ would be required to any chosen ELM mitigation or suppression method, a challenging engineering target to meet (even disregarding other drawbacks all active ELM mitigation or suppression techniques might have concerning the plasma performance). For this reason, a plasma scenario which is naturally ELM-free, as for example the QH-mode [37], the I-mode [38], or even negative triangularity $[39,40,41]$ would be extremely beneficial for a machine like EU-DEMO, whose mission includes stringent availability requirements - to a much higher extent than ITER, which is still an experiment.

Both QH-mode and I-mode have however a number of features which must be carefully considered when extrapolated to a reactor device - on top of the narrow operational space so far available in comparison to the ELMy H-mode, which is by itself a serious drawback. In particular, the main concerns linked to the adoption of the $\mathrm{QH}$ mode in a DEMO machine are:

- Difficult accessibility: a high rotational flow shear in the edge seems to be the main ingredient to access the QH-mode. It is however unclear how this rotational shear requirement translates to a DEMO reactor scale, and also how to reliably obtain it. Tangential NBI and also RMP coils [42] are possible candidates for this purpose, but both might exhibit difficulties in integration, especially if a large rotation (gradient) is needed. There may also be an impact on the allowable toroidal magnetic ripple.

- Typically observed at low density - although reasonably high Greenwald fraction have been observed during ramps [43]. This might be due to the fact that current machines are not able to operate at high density and low collisionality at the same time. Thus, this might favourably extrapolate to ITER and EU-DEMO. However, this currently prevents the community from exploring in the experiments some extremely important plasma conditions, for example a $\mathrm{QH}$-mode in presence of detached divertor.

Instead, the main concerns regarding an I-mode reactor are:

- Absence of density pedestal: in contrast to $\mathrm{QH}-$ mode, where there is some indication that the density can be recovered at DEMO scales, the absence of a density pedestal in I-mode can possibly not be compensated for in extrapolation to a reactor size. This means that, at the same machine 
radius and field, an I-mode will most likely produce less power than the corresponding $\mathrm{H}$-mode. This occurrence has also been confirmed by recent ASTRA/TGLF simulation scans around the EUDEMO working point [44].

- $\quad$ Larger threshold power than H-mode: the scaling for the LI transition power proposed by Hubbard et al. [45] indicates that the LI threshold power has a weaker dependence on the magnetic field than the LH one. Nevertheless, at EU-DEMO parameters, one still observes that $P_{L I}>P_{L H}$, as the baseline magnetic field is not sufficiently high to take advantage of the weaker dependence on $B$. This is clearly going to exacerbate the problem of reaching divertor detachment by means of seeded impurities (incidentally: as for $\mathrm{QH}$-mode, no experimental observation of detached I-mode plasmas is to our knowledge available). Furthermore, although ELMfree, the I-mode exhibits power "bursts" on the divertor target [46], whose nature is still under investigation - although they are observed to almost disappear when the plasma is operated sufficiently far away from the IH transition.

The possibility of exploring these regimes in ITER is at the moment debated. From the current understanding, it might be possible to run QH-mode shots in ITER, but Imode operation is instead questionable. This because the need of reversing the toroidal field direction (to obtain an unfavourable $\mathrm{H}$-mode access and enter I-mode first) would force a simultaneous reversing of $I_{p}$ to keep the field line helicity, and thus a proper alignment with the divertor tiles. This in turn would cause the NBI to operate in counter-current. Also, the ITER NB is not required to work with reversed field. In general, it seems difficult that an optimised DEMO will be able to work with both field directions. Thus, the impact of I-mode on the machine layout shall be carefully evaluated in advance. A more thorough analysis of the strategy to validate $\mathrm{QH}$-mode and I-mode applicability to DEMO has been recently carried out by an EUROfusion ad-hoc group [48].

Clearly, no validation of negative triangularity L-mode can be expected from ITER, as it would require large modifications to the whole machine architecture. This plasma configuration has recently received great attention from the fusion community, as it seems to be able to combine the robustness of L-mode with the confinement capability of an H-mode [47 and references therein]. Such a solution would be extremely attractive for a reactor, if these features are confirmed by future investigations. Still, the question about which machine can validate such scenario at reactor relevant parameters before a DEMO is built remains open, as no negative triangularity equivalent of ITER is at the moment foreseen.

\section{Conclusions}

The role of ITER in the development of nuclear fusion as an energy source is of paramount importance, as only in ITER the exploration of reactor relevant, dominantly alpha heated plasmas can be performed. Nevertheless, the first attempts of designing an EU-DEMO, a machine charged of producing a net electrical power and of demonstrating the robustness of fusion as an energy source, have pointed out various aspects where the currently adopted ITER solution is not applicable. In the present paper, the most relevant differences related to the plasma scenario have been discussed. Firstly, the role and the difficulties linked to the introduction of core radiation obtained by means of seeded impurities in EUDEMO has been analyzed. Secondly, it has been acknowledged that a fast plasma termination with loss of plasma control at high current poses high risks on the PFC integrity in EU-DEMO, and thus other means to protect the PFCs during current ramps, as for example divertor sweeping when divertor detachment is lost, have to be considered in the machine design. Thirdly, the ITER solution for the control of the so-called sawteeth instabilities (ST) has been shown not to be attractive for EU-DEMO by virtue of the excessively high control power requirements. Other solutions have thus been explored, and their consequences assessed. Finally, the problem of protecting the PFCs from ELMs has recently oriented the interest of the fusion community on naturally ELM-free regimes, like QH-mode, I-mode and negative triangularity, thus deviating from the ITER baseline ELMy H-mode with ELM-mitigation. However, for these ELM-free scenarios, which at this stage are poorly explored and understood, the open question is whether there is a possibility to have a reliable scenario validation at reactor relevant parameters in ITER and, if not, on which machine this can take place in order to avoid a risky extrapolation from the existing small devices to a DEMO scale.

Finally, it is worth to mention that EU-DEMO, as well as ITER, urgently needs a reliable strategy to prevent disruptions or at least to largely mitigate their consequences. Strictly speaking, this is not a challenge "beyond ITER" (and for this reason it has not been addressed in this work) but a common goal for the two devices, whose assessment is of high importance for the future of fusion energy research. The design solution to protect the first wall from disruptions in EU-DEMO is described in [49].

\section{Acknowledgments}

This work has been carried out within the framework of the EUROfusion Consortium and has received funding from the Euratom research and training programme 2014-2018 and 2019-2020 under grant agreement No 633053. The views and opinions expressed herein do not necessarily reflect those of the European Commission. 


\section{References}

[1] European Research Roadmap to the Realisation of Fusion Energy

[https://www.eurofusion.org/fileadmin/user_upload/EURO fusion/Documents/2018_Research_roadmap_long_version _01.pdf]

[2] Federici G. et al., 2019 Nucl. Fusion 59, 066013.

[3] Kovari M. et al., 2014 Fusion Eng. and Design 893054

[4] Kovari M.et al., 2016 Fusion Eng. and Design. 1049

[5] Federici G. et al., 2014 Fusion Eng. and Design 89882

[6] Aymar R. et al., 2002 Plasma Phys. Control. Fusion 44 519

[7] Green B. J. and ITER International Team and Participant Teams, 2003 Plasma Phys. Control. Fusion 45687

[8] Pereverzev G.V., 1991 IPP Report 5/42

[9] Pereverzev G.V. and Yushmanov P.N., 2002 IPP Report $5 / 98$

[10] Fable E. et al., 2013 Plasma Phys. Control. Fusion 55 124028

[11] Wagner F et al., 1982 Phys. Rev. Lett. 491408

[12] Uckan N.A. 1989 ITER physics design guidelines, ITERTN-PH-8-6

[13] Loarte A. et al., 2007 Nucl. Fusion 47 S203

[14] Wenninger R. et al 2017 Nucl. Fusion57 046002

[15] Janky F. et al., 2017 Fusion Eng. and Design 123, 555

[16] Franke Th. et al., subm. to Fusion Eng. Design (ISFNT 2019)

[17] Maviglia F. et al., 2018 Fusion Eng. Des, 136410

[18] Bachmann C. et al., accepted by Fusion Eng. Des.

[19] Maviglia F. et al., 2018 Fusion Eng. Des. 136, 410

[20] You J.H. et al., 2016 Nuclear Materials and Energy 9171

[21] Siccinio M. et al., 2019 Nucl. Fusion 59, 106026

[22] Maviglia F. et al., 2016 Fusion Eng. Des. 1091067

[23] Felici F. et al., 2011 Nucl. Fusion 51, 083052

[24] Teplukhina A. A. et al, 2017 Plasma Phys. Control. Fusion 59, 124004

[25] Lehnen M. et al., 2015 Journal of Nucl. Mat. 46339

[26] Lehnen, M., et al., 26th IAEA Fusion Energy Conference.

[27] Leonard A. W., 2018 Plasma Phys. Control. Fusion 60, 044001

[28] Reinke M. L. et al., 2017 Nucl. Mat. and Energy 12, 91

[29] Henderson S. S. et al., 2018 Nucl. Fusion 58, 016047

[30] Verhaegh K. et al., 2017 Nucl. Mat. and Energy 12, 1112

[31] Chapman I. et al., 2010 Nucl. Fusion. 50102001

[32] Goodman T. P. et al., 2011 Phys. Rev. Letters 106, 245002

[33] Porcelli F. et al., 1996 Plasma Phys. Control. Fusion 38, 2163

[34] Lerche E. et al., 2017 Nucl. Fusion 57036027

[35] Eich Th. et al., 2017 Nuclear Materials and Energy 12, 84

[36] Raffray A.R. and Federici G., 1997 J. Nucl. Mater. 244, 85

[37] Burrell K. H. et al., 2002 Plasma Phys. Control. Fusion 44, A253

[38] Whyte D. et al., 2010 Nucl. Fusion 50, 105005

[39] Camenen Y. et al., 2017 Nucl. Fusion 57, 086002

[40] Medvedev S. Yu. Et al., 2015 Nucl. Fusion 55, 063013 (2015)

[41] Austin M. et al.,2019 Phys. Rev. Letters 122115001
[42] Garofalo A. M. et al., 2011 Nucl. Fusion 51, 083018

[43] Solomon W. M. et al., 2015 Nucl. Fusion55 073031

[44] Palermo F. et al., 2019 Nucl. Fusion59 096010

[45] Hubbard, A. E. et al., 2017 Nucl. Fusion 57. 126039

[46] Happel T. et al., 2017 Plasma Phys. Control. Fusion 59 014004

[47] Kikuchi M. et al., 2019 Nucl. Fusion59 056017

[48] Final Report - EF ad-hoc group on ELM-free regimes https://idm.euro-fusion.org/?uid=2NF9QF

[49] Maviglia F. et al., subm. to Fusion Eng. Design (ISFNT 2019) 